\title{
The life and work of E. C. George Sudarshan
}

\author{
N. Mukunda* \\ INSA Distinguished Professor, Indian Academy of Sciences, Bengaluru 560 080, India
}

E. C. G. Sudarshan is widely regarded as the most gifted theoretical physicist of Indian origin in the latter half of the 20th century. This article describes his early student years in India, and at the Tata Institute of Fundamental Research in Bombay, before he left for USA in 1955 to work with R. E. Marshak at the University of Rochester. It then recounts his career as it evolved thereafter, and his decision to settle there. His contributions in many areas of physics, in each of which he made a distinct mark, are recalled. In particular, his work on the $V-A$ theory of the weak interactions, and on the Diagonal Representation in quantum optics, are described in some depth and detail. Sudarshan maintained strong links with the Indian physics and scientific communities all his life. From the 1970s onwards, he was at the Indian Institute of Science in Bangalore, and then at the Institute of Mathematical Sciences in Madras, till 1990. The events of this period are recounted. Some remarks on his personality, and his views on life and philosophy, conclude the article.

\section{Introduction}

Modern science came to India, through teaching at College and University levels, only in the late 1800s. In those days, in a handful of institutions across the country, there were a few truly dedicated teachers, many from the Jesuit and other Christian missionary orders. The three earliest modern universities at Calcutta, Bombay and Madras were all established in 1857; however, their role was largely as examining bodies. Given this, it is remarkable that the idea of promoting research in science was thought of quite soon, the Indian Association for the Cultivation of Science being set up in Calcutta (now Kolkata) by Mahendra Lal Sircar already in 1876.

Despite this relatively late beginning, Indian contributions in theoretical physics (to which we limit ourselves) have been remarkable, many of them achieving worldwide impact and acclaim. The pioneers were Meghnad Saha (1893-1956) and Satyendra Nath Bose (18941974), both of whom had their education and did their outstanding work in the India of their times. Later came Homi Jehangir Bhabha (1909-1966) and Subrahmanyam Chandrasekhar (1910-1995), who both went to Cambridge in England for their doctoral work in the early

*e-mail:nmukunda@gmail.com 1930s. Of them, only the former came back to India (in 1939), and after a few years of devoting himself to research in theoretical (and some experimental) physics at the Indian Institute of Science (IISc) in Bangalore, he turned to institution-building on an impressive scale.

Two outstanding theoretical physicists in the next phase, Prahlad Chunilal Vaidya (1918-2010) and Amal Kumar Raychaudhuri (1923-2005), both worked in the area of general relativity. They were trained entirely within the Indian university system, to which they contributed throughout their lives. The mathematician HarishChandra (1923-1983), originally a physicist, belonged to the same generation.

The next important names from this region are Abdus Salam (1926-1996) and E. C. G. Sudarshan (1931-2018). Both were born in pre-independence times, and lived and studied in India up to College and University levels. Thereafter, Salam (like Bhabha and Chandrasekhar) went to Cambridge in 1946, completed his $\mathrm{PhD}$ in 1951, and after an unsuccessful attempt to return to Pakistan, went back to the West. His life was spent in England and Italy.

This article is devoted to Sudarshan. An attempt is made to describe his life and important influences on him up to the time he went abroad in 1955; his career in USA, and his work and major accomplishments in physics. He was a highly original and in some ways unusual physicist, with a remarkably broad range of interests and gifts. His work had tremendous impact in several areas, though unfortunately he did not receive proper credit for all his achievements.

Sudarshan retained strong links with the Indian physics and larger scientific community all his life, even though he was primarily a full time academic based in USA.

This article will try to convey all this as objectively as possible, bringing out also, to some extent, aspects of his personality.

\section{Early life and education}

Ennackal Chandy George (ECG) was born into a Syrian Christian family on 16 September 1931 in Pallam, Kerala. His mother Achamma was a school teacher, and father E. I. Chandy a revenue inspector in the old Travancore state. He was the second of three boys, between Joseph and Thomas Alexander.

ECG's aptitude for mathematics was apparently evident from his school days. After high school, he completed the two-year Intermediate at the Church Missionary Society 
(CMS) College in Kottayam in 1948. This college, established in 1817, was the oldest such institution in Kerala.

From CMS College, ECG went to the Madras Christian College (MCC) in Madras (now Chennai) for his B Sc (Honours) in Physics. This was from 1948 to 1951, after which he stayed on for a year as a demonstrator in physics. He said later that the course on classical optics taught by M. A. Thangaraj was a wonderful experience. Among his contemporaries at MCC were P. M. Mathews (born 1932) and R. Srinivasan (born 1931), long settled in Chennai and Mysore respectively. In 1952, by lapse of time, ECG received the MA degree of the University of Madras.

ECG then joined the Tata Institute of Fundamental Research (TIFR) in Bombay (now Mumbai) in the spring of 1952 as a research student. TIFR had been set up by Bhabha in 1945, and soon became a leading research institution in the country. One of ECG's fellow students, to whom he remained close all his life, was Ramanuja Vijayaraghavan (born 1931) who worked at TIFR for his entire career. Vijayaraghavan's area of research was experimental condensed matter physics; after retirement he divides his time between Mumbai and Singapore. The three years, 1952 to 1955 , spent by ECG at TIFR turned out to be enormously important in many ways. He was initially asked to do experimental work in particle physics, using the photographic emulsion technique. In this phase he worked closely with Sukumar Biswas (1924-2009) and Ranjit Roy Daniel (1923-2005), as well as with Bernard Peters (1910-1993), all well-known cosmic-ray physicists. Later he was able to turn to theoretical work, developing his true interests and strengths. These years brought him close to two other senior physicists at TIFR - the condensed matter theorist Kundan Singh Singwi (1919-1990), and the experimental nuclear physicist Raja Ramanna (1925-2004).

Then in late 1954, the legendary Paul A. M. Dirac (1902-1984), a teacher of Bhabha at Cambridge in the

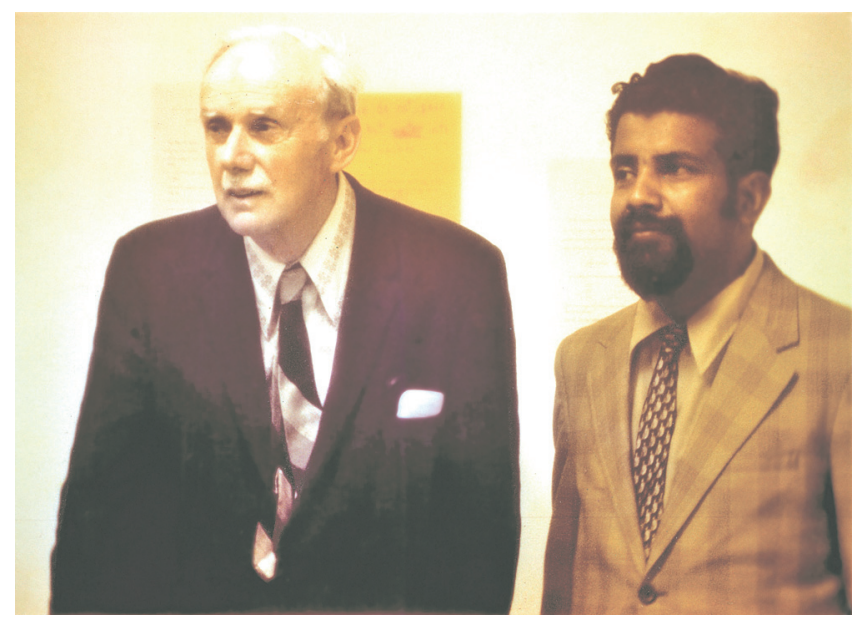

Paul Dirac with ECG at Austin, Texas, Spring 1973 1930s, visited TIFR and gave a course of lectures on quantum mechanics. The lecture notes were prepared by ECG and K. K. Gupta, a student of Bhabha. This work brought ECG and Dirac into close contact - a unique and unimaginably fortunate opportunity to learn the subject from the master himself. It is reasonable to imagine that this experience must have shaped ECG's attitude to quantum mechanics all his life, giving him the daring and courage to push and test the principles of the subject in many directions. ECG greatly admired Dirac, and the two remained lifelong friends.

Another important earlier visitor to TIFR in this period, in August 1953, was Robert Eugene Marshak (1916-1992), who had been a student of Hans Bethe at Cornell University, USA in the late 1930s. Marshak was a leading theoretical elementary particle physicist, a contemporary of Julian Schwinger (1918-1994) and Richard Phillips Feynman (1918-1988) (all three from in and around New York city), and a good friend of Bhabha. He had created a vibrant group at the University of Rochester, USA, with talented students brought in from many parts of the world. During his visit and lectures, Marshak was so impressed by ECG that he persuaded him to come to Rochester and work with him for his $\mathrm{PhD}$. After some initial difficulties, ECG was able to go to Rochester in September 1955.

Some time before this, in 1954, ECG and Lalitha (neé Rau), another student at TIFR, were married. It was at this time that he added the Hindu name 'Sudarshan'; so ECG became ECGS. They were to have three sons: Pradeep Alexander (born 1959); Arvind Jewett (1962-2004); and Ashok John (born 1966). The marriage lasted till 1989. Sometime soon after, Gopalakrishnan Bhamathi (born 1938) and ECG were married.

\section{The US years - Rochester, Harvard, Syracuse and Austin}

At the time ECG reached Rochester, there was enormous excitement in the field of elementary particle physics, largely on account of experimental results using the photographic emulsion technique which he had learnt at TIFR. Many new particles had been discovered in the cosmic radiation - the $\pi$ mesons, 'strange' particles like the $\mathrm{K}$ mesons, and the hyperons $\Lambda, \Sigma, \ldots$ (heavier 'cousins' of the proton and neutron). The production and study of these particles at accelerators was yet to come. Their decay modes and characteristics had led to intriguing puzzles, one of the best known being the so called ' $\tau-\theta$ puzzle'. (The $\tau$ meson had been discovered in 1953 by M. G. K. Menon (1928-2016) and collaborators working at Bristol, UK, using the photographic emulsion method to study cosmic rays.) In 1956, as a way out of these problems, T. D. Lee (born 1926) and C. N. Yang (born 1922) suggested that spatial inversion may not be a valid symmetry of nature in these processes ${ }^{1}$. Parity may 
not be conserved in the weak interactions. Their careful analysis revealed that this had never been experimentally tested, and they suggested that this be done in $\beta$-decays. The definitive experiment was carried out by Lee's Columbia colleague Mme C. S. Wu (1912-1997) and collaborators at the National Bureau of Standards in Maryland, USA, and the results were announced in 1957 (ref. 2). Indeed, parity was not conserved in weak processes. Lee and Yang shared the 1957 Nobel Prize in Physics ${ }^{3}$.

The original 1933 Fermi (1901-1954) theory of $\beta$ decay had of course assumed that parity was conserved ${ }^{4}$. In 1936, a variation of the Fermi theory had been made by G. Gamow (1904-1968) and E. Teller (1908-2003), again conserving parity ${ }^{5}$. After the events of 1956-1957, it became a major problem to determine the correct form of a universal Fermi interaction to replace Fermi's originally formulated 1933-34 theory, capable of describing $\beta$-decay as well as all strange particle decays, incorporating parity violation. It was to this extremely active field that, most fortunately, Marshak introduced ECG to work for his Ph D. By early 1957, after an extensive study of all existing experimental results and allowed theoretical possibilities, ECG and Marshak had arrived at the so-called ' $\mathrm{V}-\mathrm{A}$ ' structure for the universal Fermi interaction as the only tenable hypothesis. We discuss this work later, allowing for some repetitions.

ECG completed his Ph D in 1958. Some of his fellow students were Sudhir Pandya (born 1928) from India graduating in 1957 in nuclear theory and later living and working in India; Prabhakar Pandurang Kane (born 1929), Ph D 1958, who also worked later in India; Susumu Okubo (1930-2015) from Japan graduating under Marshak in 1958, and Tullio Regge (1931-2014) from Italy, also graduating under Marshak in 1957. Senior to them was the Italian Eduardo Caianiello (1921-1993), who had completed his Ph D under Marshak in 1950, and had set up a flourishing school in theoretical physics in Naples during the years 1956-1972.

After submitting his $\mathrm{PhD}$ thesis, ECG spent two years - 1957 to 1959 - as Research Fellow with Schwinger at Harvard University. During this period he made frequent visits to Rochester, travelling overnight by Greyhound buses, to continue collaborations with Marshak and Okubo - many papers by the trio date from these years. At Harvard he did a well-known piece of work with K. Johnson, a student of Schwinger, on inconsistencies in higher spin field theories in external fields ${ }^{6}$. This was based on Schwinger's Action Principle, and it inspired a considerable amount of work by G. Velo, A. S. Wightman and others, some years later. Following a suggestion by Schwinger, ECG also collaborated with S. Deser (born 1931) and W. Gilbert (born 1932) on problems in axiomatic quantum field theory - integral representations for two-point and three-point functions. Gilbert had been a student of Salam in physics at Cambridge, in 1980 he shared the Nobel Prize in Chemistry with F.
Sanger and P. Berg. ECG had apparently planned to return to India after the two years at Harvard. In a paper with Deser and Gilbert submitted to the Physical Review on 15 July 1959, ECG's name was accompanied by a footnote 'On leave of absence from the Tata Institute of Fundamental Research, Bombay, India'.

However, soon after, Marshak offered him a position; and though ECG met Bhabha at Washington, the decision had been made to stay on in the US. In a paper with P. M. Mathews and Jayaseetha Rau submitted to the Physical Review on 15 August 1960, there was no mention of TIFR ${ }^{7}$.

In 1959, ECG returned to Rochester and joined the physics faculty as Assistant Professor; two years later he became Associate Professor. His early students in these years - a large group with an international flavour - were R. Acharya (1936-2018) from India; Thomas Jordan (1936-2018), Douglas Currie, Korkut Bardakci from Turkey; M. Y. Han (1934-2016) from South Korea and M. E. Arons, Gabriel Pinski and N. Mukunda (also from India). In 1962, ECG taught the graduate classical mechanics course in an unusual way, stressing the importance of Lie groups and Lie algebras both in the general formalism and in the actions of space-time and other symmetry groups. In hindsight one can see the influence of Schwinger's style in this treatment of a venerable old subject. (Some years later, in 1974, this led to the book Classical Dynamics - A Modern Perspective co-authored with N. Mukunda ${ }^{8}$.) It was in 1961 that his fundamental work with P. M. Mathews and J. Rau titled 'Stochastic dynamics of quantum mechanical systems' appeared. This was in a sense far ahead of the times, heralding the quantum theory of open systems which is today at the base of quantum information theory.

In 1963, ECG discovered the Diagonal Coherent State Representation for arbitrary states of quantum optical fields. This is discussed in more detail later, especially the vexed problem of how to apportion the credit between him and R. J. Glauber (born 1925). The two outstanding faculty members at Rochester at that time, in the field of optics, were Emil Wolf (1922-2018) and from 1964, Leonard Mandel (1927-2001); they were instrumental in creating the conditions leading to ECG's work, and later collaborated with him. On the mathematical side, this work involved the use of 'distributions' of an extremely singular nature. We are reminded of the Dirac 'delta function', introduced as early as 1930 by Dirac in his formulation of quantum mechanics. For many years, this 'function' was not accepted by the mathematics community. It was only in 1944-45 that the theory of distributions by Laurent Schwartz (1915-2002) provided a satisfactory mathematical framework able to handle rigorously the Dirac delta function (and finitely many derivatives of it). However, in ECG's work there appeared many more singular objects, distributions whose Fourier transforms could be diverging Gaussian expressions, which even Schwartz's theory could not handle. The 
courage, indeed daring, to use such mathematical objects, one imagines, may have had its roots in his discussions with Dirac at TIFR in 1954.

Another important work published in 1963 was with Currie and Jordan, leading up to the No Interaction Theorem for classical relativistic Hamiltonian theories of point particles ${ }^{9}$. Many years later, 1980-1984, these problems were re-examined in the framework of Dirac's constrained Hamiltonian dynamics, as part of a large collaborative effort that lasted from 1979 to 1984.

ECG took sabbatical leave in 1963-64, spending the first half at the University of Bern, and the second at Brandeis University. In between he also visited the Institute of Mathematical Sciences (IMSc) in Madras in India. At Bern he gave a set of lectures on quantum optics; the notes by F. Ghielmetti served as the basis for the 1968 book Fundamentals of Quantum Optics with J. R. Klauder (born 1932) ${ }^{10}$. This is one of the early books on the subject, and it dealt with the 1963 diagonal coherent state representation in all its mathematical detail. At IMSc ECG introduced Lochlainn O'Raifeartaigh (1933-2000), who was also visiting, to the problem of combining internal symmetry with relativistic invariance. (In fact, ECG had met O'Raifeartaigh in Europe a few months earlier and suggested that he visit IMSc.) This was an active area of research at that time, and a paper from Brandeis in 1964 by Schnitzer, Mayer, Acharya, Sudarshan and Han (evidently inspired by ECG) was referred to by O'Raifeartaigh as the SMASH paper.

In 1964, ECG moved from Rochester to Syracuse University as Professor, and created a group in elementary particle physics. Up to that time the major emphasis at Syracuse had been on general relativity led by Peter G. Bergman (1915-2002), once an assistant to Albert Einstein in Princeton. ECG's initial colleagues were O'Raifeartaigh, A. P. Balachandran (born 1938) and A. J. Macfarlane. As a student, Jacob G. Kuriyan from India, who had come to Rochester in 1963, moved with ECG to Syracuse and completed his $\mathrm{PhD}$ in 1967. In 1965, Sandip Pakvasa (born 1935; Ph D 1965 from Purdue University), a leader in weak interactions and neutrino physics today, came as a postdoctoral fellow and became a highly valued lifelong professional colleague. Thanks to the links with the Napoli group, several students and visiting faculty from there also came to work at Syracuse. In 1967, Joseph M. Schechter (1965 Rochester Ph D with Okubo) joined the group.

Probably the best known result from ECG's group in these years at Syracuse is the O'Raifeartaigh Theorem showing the impossibility of combining internal symmetry and relativistic invariance in a nontrivial manner.

In 1969, ECG made his last move within the US from Syracuse to the University of Texas at Austin, as Professor and Co-Director (with Yuval Ne'eman) of the Centre for Particle Theory. (His successor at Syracuse was Kameshwar C. Wali (born 1927), a theoretical elementary particle physicist, who moved from the Argonne National Laboratory.) He remained in Austin for the rest of his professional life in the US, teaching and directing and doing research in theoretical physics. Among the areas he explored in almost five decades, there were relativistic wave equations, indefinite metric quantum field theories using the concept of shadow states, the Zeno paradox and effect in quantum mechanics, quantum mechanics of open systems, the measurement problem in quantum mechanics, and supersymmetry in particle physics. In addition, there were long-term collaborations with colleagues in Italy and Spain, and in India. Two of his students from the Austin years live and work in India: Urjit A. Yajnik (1982-1986) at the Indian Institute of Technology, Bombay, and Anil Shaji (1999-2005) at the Indian Institute of Science Education and Research, Trivandrum.

It is appropriate to recall that during his long working life in the US, ECG had particularly cordial relationships with two outstanding Japanese theoretical physicists: Hiroomi Umezawa (1924-1995) and Yoichiro Nambu (1921-2015). Umezawa was a very original quantum field theorist, who moved from Napoli to the University of Wisconsin at Milwaukee, then onto the University of Alberta at Edmonton, with some assistance from ECG. Nambu worked at the University of Chicago and won the Nobel Prize in 2008. ECG's student Han collaborated with Nambu in the construction of the Han-Nambu quark model in 1965. And in 1982, Nambu attended a conference in Bangalore to celebrate 25 years of the Sudarshan-Marshak V - A theory of 1957.

\section{Events and publications from 2006}

In 2006, ECG turned 75. In celebration, an international conference and a symposium were held, and a book published. The book and the two proceedings are: (i) E. C. G. Sudarshan-Selected Scientific Papers (ed. Nair, R.), Centre for Philosophy and Foundations of Science, New Delhi, 2006. (ii) Particles and fields: classical and quantum. Journal of Physics: Conference Series, 2007, 87. (iii) Sudarshan: seven science quests. Journal of Physics: Conference Series, 2009, 196.

The book (i) contains 57 papers selected by ECG, preceded by: Autobiographical Notes; a 1985 preprint from IMSc, Chennai, by him titled 'Mid-century adventures in particle physics'; 'Origins of the universal V - A theory' by ECG and R. E. Marshak, based on a 1984 international conference talk by Marshak at Racine, Wisconsin; and 'The pain and the joy of a major scientific discovery', the text of Marshak's banquet talk at a 1991 celebration of ECG's 60th birthday.

Publication (ii) contains some of the contributions to the conference 'Particles and Fields: Classical and Quantum' held at Jaca, Spain, during 18-21 September 2006, organized by ECG's Italian and Spanish collaborators. A 'Laudatio for E. C. G. Sudarshan' by Luis J. Boya, and 
ECG's 'A glance back at five decades of scientific research' are followed by articles from the speakers.

Publication (iii) contains the Proceedings of the Symposium 'Sudarshan: Seven Science Quests' held at the University of Texas at Austin, during 6 and 7 November 2006, with an interesting structure. Apart from opening and closing messages, ECG's work was surveyed by several speakers in seven technical sessions, each devoted to one of his quests: V-A; symmetry; spin and statistics; quantum coherence; quantum Zeno effect; Tachyons, and open systems. While the work in each of these streams is important, it would be out of place to dwell in detail on all of them here. We have earlier mentioned ECG's work with Currie and Jordan in the area of symmetry - the no interaction theorem in classical relativistic Hamiltonian particle mechanics. Even before this, following a discussion among Salam, ECG and Marshak, the first ever use of broken symmetry in particle physics to obtain consequences for masses and magnetic moments of the $\Sigma$-hyperons was made by Marshak, Okubo and ECG in 1957 (ref. 11). In later years, ECG and collaborators applied symmetrybased arguments to tackle several problems in classical wave optics and the kinematics of quantum mechanics.

In the area of the spin-statistics connection, the original 1940 work by Wolfgang Pauli (1900-1958) was based on Special Relativity and essentially limited to the free field case. ECG maintained always that an alternative approach based on nonrelativistic quantum mechanical notions and the Action Principle, and allowing for interactions, was what was really needed. His work on Tachyons was an attempt to show that the usually stated postulates of Special Relativity permit the existence of particles with space like energy-momenta and speeds always greater than that of light; but for them under homogeneous Lorentz transformations the processes of emission and absorption can get interchanged. Up to now there is no experimental evidence for the existence of such particles.

\section{The Quantum Zeno Effect}

This subject apparently has a long history going as far back as von Neumann's treatment of the mathematical foundations of quantum mechanics in 1932. In the traditional probabilistic interpretation of quantum mechanics, and von Neumann's treatment of the measurement process, there are several important features:

(i) All probabilities (for transitions from one state to others, as well as for survival of an initial state) are squared moduli of corresponding complex probability amplitudes.

(ii) The equation of motion of quantum mechanics, the linear Schrödinger wave equation, is obeyed in between measurements.

(iii) When a measurement is carried out, that equation is temporarily suspended, and in its place we have a collapse of the wave function.
Thus every measurement acts as an interruption of unitary Schrödinger evolution.

It is well known that for short times $\Delta t$, the probability that a given initial state $\left|\psi\left(t_{0}\right)\right\rangle$ remains unchanged, i.e. undecayed, at time $t_{0}+\Delta t$ is unity minus a quantity quadratic in $\Delta t$; equally well, the probability of survival of the initial state is close to a Gaussian rather than to an exponential:

$$
\left|\left\langle\psi\left(t_{0}\right) \mid \psi\left(t_{0}+\Delta t\right)\right\rangle\right|^{2} \simeq \exp -\left((\Delta t)^{2} / \tau^{2}\right), \text { some } \tau .
$$

In this sense, probabilities of transitions from $\left|\psi\left(t_{0}\right)\right\rangle$ to other states cannot be proportional to $\Delta t$, and thus cannot be described by transition rates. Following Dirac, in the usual presentations of quantum mechanics, if we assume we have a continuum of final states into which transitions could take place, one can by a judicious set of approximations obtain an exponential form for survival probabilities, as in radioactive decay. (It is in this way that we arrive at the Fermi Golden Rule for transition rates caused by a perturbation.) In the absence of these conditions, we do not obtain the exponential law at all.

In 1977, Baidyanath Misra (born 1937) and ECG showed that indeed all this can have observable consequences $^{12}$. In particular, if one checks sufficiently frequently whether the initial state $\left|\psi\left(t_{0}\right)\right\rangle$ has not decayed, then as this frequency is increased indefinitely, $\left|\psi\left(t_{0}\right)\right\rangle$ will not decay at all. And in the case of an unstable particle or state, sufficiently frequent measurement inhibits the decay and converts instability to stability.

The original analysis by Misra and ECG is mathematically quite sophisticated. Over the years it has been generalized, in particular by ECG and his Italian collaborators G. Marmo, S. Pascazio and P. Facchi. Experiments by W. M. Itano, and by M. Raizen, and their collaborators, have confirmed the existence of the Quantum Zeno Effect.

\section{Quantum theory of open systems}

As mentioned earlier, the basic equation of motion in quantum mechanics is the Schrödinger wave equation. This involves the energy of the system in the form of the Hamiltonian operator $\hat{H}(t)$; it is an equation obeyed by the complex wave function $\psi(t)$ corresponding to the time-dependent state of the system:

$$
i \hbar \frac{\mathrm{d}}{\mathrm{d} t} \psi(t)=\hat{H}(t) \psi(t)
$$

Properly speaking, a wave function describes a so called 'pure state', a state with maximum possible information, and the wave equation is valid for a closed or isolated quantum system.

As early as in 1961, along with P. M. Mathews and Jayaseetha Rau, ECG envisaged a two-fold generalization of this situation ${ }^{13}$. General mixed or 'impure' states of the 
system are described by so called 'density matrices' $\rho(t)$, which may be pictured as being 'quadratic' in wave functions, equally well as a classical statistical ensemble of pure states:

$$
\rho(t) \sim \psi(t) \psi(t)^{*}, \rho(t)=\sum_{r} p_{r} \psi_{r}(t) \psi_{r}(t)^{*}
$$

And the most general conceivable time evolution of such states, preserving the defining properties of density matrices, was termed as 'stochastic quantum dynamics'. The law of such evolution was taken to be linear in the density matrix. The motivation of this work lay in the general classical theory of stochastic processes, and a desire to generalize it to the quantum domain to serve as the starting point or foundation for the more phenomenological Pauli master equation in quantum statistical mechanics.

The density matrix in quantum mechanics generalizes in a profound way the classical idea of a probability distribution; therefore it retains as one of its basic properties, the idea of being non-negative. Any dynamical map must preserve this and other properties of density matrices; we call this the positivity of the map itself.

It turned out that the situation is much more subtle and profound than ECG and co-authors initially appreciated. Since we are dealing in general with open quantum systems, there is always the possibility that the system being studied is part of a larger quantum system. The possible presence of quantum correlations between parts of a larger quantum system leads to a genuinely new requirement on dynamical maps - they have to be completely positive, or possess the CP property. This goes beyond the previously mentioned positivity property, because of the subtlety of quantum correlations.

In his Autobiographical Notes of 2006, ECG has said that the existence and importance of the $\mathrm{CP}$ condition was not initially appreciated by him. It took many years to unravel all the properties of dynamical maps, and to set up a robust formalism for handling open quantum systems. Along the way the Italian physicist Vittorio Gorini joined ECG in this work in the 1970s; then they made contact and collaborated with the Polish mathematical physicist Andrej Kossakowski. As a result of this sustained attack on a genuinely difficult problem with many algebraic features, a framework for describing the most general physically acceptable dynamical map, and a form for the corresponding evolution equation, was finally obtained. At some point Gorini discussed this work and ideas with G. Lindblad in Sweden, who had been working on Kossakowski's results. As these efforts progressed, it was realized that the mathematician W. F. Stinespring had dealt with complete positivity as far back as in 1955; and M. D. Choi had obtained important related results in 1975 .

Today the final form of the evolution equation for open quantum systems, incorporating the $\mathrm{CP}$ property, is named after all of them - ECG, Gorini, Kossakowski, Lindbladand one more, Kraus! Here is what it looks like:

$$
\begin{aligned}
i \hbar \frac{\mathrm{d}}{\mathrm{d} t} \rho(t) & =[\hat{H}(t), \rho(t)] \\
& +\frac{i}{2} \sum_{j}\left(2 A_{j} \rho A_{j}^{+}-A_{j}^{+} A_{j} \rho-\rho A_{j}^{+} A_{j}\right),
\end{aligned}
$$

where $\hat{H}(t)$ is the Hamiltonian and the $A_{j}$ are additional operators.

An excellent and detailed account of these developments is contained in ref. 14 .

\section{The $\mathrm{V}-\mathrm{A}$ and diagonal representation stories}

Of all his work in varied fields, two of ECG's achievements are extremely highly regarded, with many considering that they are of the Nobel class. We have referred to them previously, here we give a more detailed discussion. Borrowing from a phrase of Albert Einstein, we shall be 'as brief as possible, but not more so.'

\section{The case of $V-A$}

The phenomenon of radioactivity, discovered in 1896 by Henri Becquerel, comes in three forms which were recognized by Ernest Rutherford. The $\beta$ form is the second of them. The basic or primitive $\beta$ processes (until the 1940 s and 1950s) are the decay of the neutron into a proton, an electron and an (anti) neutrino, and the reverse 'proton decay' (possible only within a nucleus) into neutron, positron and neutrino:

$$
n \rightarrow p+e^{-}+\bar{v}_{e}, p \rightarrow n+e^{+}+v_{e} \text { (inside a nucleus). }
$$

(Here $v_{e}$ is the electron-type neutrino; later two others have been found.) The existence of the neutrino had been proposed by Wolfgang Pauli (1900-1958) in 1930, in order to restore conservation of energy, momentum and angular momentum in $\beta$-decays in which it was known that the electron is emitted with a continuous energy spectrum $^{15}$. The monumental Fermi theory of $\beta$-decay was proposed in 1933, just a year after the discovery of the neutron by James Chadwick in 1932. The most important experimental results available to Fermi were from the work of B. W. Sargent in 1932-33.

Fermi's theory gave quantitative expression to a new fundamental force or interaction in Nature, namely the Weak Interaction, entirely within the framework of quantum mechanics. He used the neutrino hypothesis and the formalism of quantum field theory (QFT), which as quantum electrodynamics (QED) had been proposed by Dirac in 1927 to treat electromagnetic interactions. Fermi had reviewed QED in exquisite fashion in the Reviews of 
Modern Physics in 1932 (ref. 16). Thus his 1933 work was the second major use of QFT methods in physics, and in turn it inspired Hideki Yukawa's (1907-1981) work on the meson theory of nuclear forces in 1935 (ref. 17).

In the Fermi theory the basic 'variables' are the four quantum fields corresponding to the proton, the neutron, the electron and the neutrino, each a four-component Dirac field. Fermi assumed they interact multiplicatively and locally at each point in space-time. He also assumed both Lorentz invariance and space inversion symmetry, i.e. conservation of parity. By a great leap of intuition based on the case of QED, he chose the vector - V-form for the interaction - a Lorentz four-vector formed out of the proton and neutron fields was contracted with a similar four-vector formed from the electron and neutrino fields. Thus the interaction term in the Lagrangian density in Fermi's theory was (in modern notation) the four-fermi expression

$$
\begin{aligned}
\mathcal{L}_{\text {Fermi }}(x)= & -\frac{G_{F}}{\sqrt{2}} \bar{\psi}_{p}(x) \gamma^{\mu} \psi_{n}(x) \bar{\psi}_{e}(x) \gamma_{\mu} \psi_{\nu}(x) \\
& + \text { hermitian conjugate. }
\end{aligned}
$$

Here $G_{\mathrm{F}}$ is the Fermi coupling constant, known to have the value $(\hbar c)^{3} \times 1.166 \times 10^{-5}(\mathrm{GeV})^{-2}$; and $\gamma_{\mu}$ are the Dirac $4 \times 4$ gamma matrices.

With this interaction structure, and working to lowest nontrivial order of perturbation theory, Fermi was able to explain the principal experimental results on $\beta$-decay put together by Sargent. Fairly soon, two things became evident: (i) the theory did not permit meaningful calculations to higher orders in perturbation theory; (ii) the $\mathrm{V}$-form chosen by Fermi was one of five forms permitted by relativity and parity conservation, the four others being $\mathrm{S}$ (scalar, $\gamma^{\mu} \rightarrow 1$ ), $\mathrm{T}$ (tensor, $\gamma^{\mu} \rightarrow \frac{1}{2}\left[\gamma^{\mu}, \gamma^{\nu}\right]$ ), A (axial vector, $\gamma^{\mu} \rightarrow \gamma_{5} \gamma^{\mu}$ ) and $\mathrm{P}$ (pseudoscalar, $\gamma^{\mu} \rightarrow \gamma_{5}$ ). Here, $\gamma_{5}=\gamma_{0} \gamma_{1} \gamma_{2} \gamma_{3}$; and in each case the replacement for $\gamma^{\mu}$ in eq. (6) is indicated. Thus, in principle, there could be five terms in $\mathcal{L}_{\text {Fermi }}(x)$, labelled $\mathrm{S}, \mathrm{V}, \mathrm{T}, \mathrm{A}, \mathrm{P}$ all added together and each with its own coupling constant.

In the Fermi theory, in the nonrelativistic limit, $\beta$ decay conserves the nuclear spin, i.e. $\Delta J=0$. In order to handle decays with $\Delta J=1$, in 1936 Gamow and Teller proposed inclusion of the $\mathrm{T}$ and $\mathrm{A}$ terms in the Fermi interaction, so we had the original Fermi V-form plus the later Gamow-Teller A-T forms.

The late 1930s, and after World War II the period from about 1947 to 1958 (and onto 1967) saw many revolutionary developments in the physics of weak interactions, indeed in the expanding field of elementary particle physics as a whole. The $\mu$ meson was discovered in 1936; and the $\pi$ meson predicted by Yukawa, in 1947 (ref. 18). These decayed via new weak processes: $\pi^{-} \rightarrow \mu \bar{v}_{\mu} ; e \bar{v}_{e} ; \mu^{-} \rightarrow e v_{\mu} \bar{v}_{e}$. (That $v_{e}$ and $v_{\mu}$ are different was established in 1962.) In addition, there was the $\mu$-capture process, basically $\mu^{-}+p \rightarrow n+v_{\mu}$. With the discoveries in the 1950 s of the unstable 'strange' particles, mesons $K^{ \pm}, K_{0}, \bar{K}_{0}$ and baryons $\Lambda, \Sigma^{ \pm}, \Sigma^{0}$, all their decays too were seen to be weak processes. Overall it became clear that there were three kinds of weak processes (mainly decays): purely leptonic ( $\mu$ decay); semileptonic (the original nuclear $\beta$-processes (5), $\pi$ decay, certain $K$, $\Lambda, \Sigma$ decays); and nonleptonic ( $K, \Lambda, \Sigma$ decays to $p, n, \pi$ only).

Gradually it emerged that these decay processes had 'strengths' similar to the original $\beta$-decays. The case of $\mu$ decay was noted in 1948 by Oskar Klein (1894-1977); that of $\mu$ capture in 1949 by J. Tiomno (1920-2011) and J. A. Wheeler (1911-2008); and of all three by T. D. Lee, M. N. Rosenbluth and C. N. Yang, also in 1949 (ref. 19). After the discoveries of the strange particles, in 1955, N. Dallaporta (1910-2003) noticed this similarity in their decays as well ${ }^{20}$. From all of these partial hints arose the idea of a Universal Fermi Interaction (UFI) underlying all these phenomena.

Then in 1954 in an incisive analysis of strange particle (nonleptonic) decays R. H. Dalitz (1925-2006) uncovered the ' $\tau-\theta$ puzzle', the apparent existence of two different particles with the same mass but decaying into final states with opposite parities ${ }^{21}$. Two years later in June 1956, Lee and Yang proposed as a solution that parity was violated in weak interactions, and that $\tau$ and $\theta$ were one and the same. They pointed out that this had never been checked directly and persuaded Lee's experimental colleague $\mathrm{Mme} \mathrm{Wu}$ at Columbia University to take up this challenge. In 1957, Wu and her colleagues demonstrated in an experiment carried out at the National Bureau of Standards in Washington DC over 2-8 January that in the $\beta$-decay of polarized ${ }^{60} \mathrm{Co}$ nuclei, the emitted electrons came out preferentially in the backward directiona clear sign of parity violation. As mentioned earlier, Lee and Yang received the 1957 Nobel Prize in Physics.

The consequences of this dramatic discovery for the Fermi theory were immediate: instead of only five possible types $-\mathrm{S}, \mathrm{V}, \mathrm{T}, \mathrm{A}, \mathrm{P}-$ of parity conserving fourfermion couplings, there were now twice as many. New parity violating forms SP, PS, VA, AV, ... could be present, each with its own strength.

It was to this extremely active and rapidly developing field that Marshak introduced ECG in late 1956, and suggested that he examine the prospects of a UFI in the new scenario. The latter plunged into the problem immediately and made amazingly rapid progress. Since the background has been adequately presented, the events of the subsequent months will be described essentially in chronological order.

(a) ECG studied all the available experimental weak interaction data in great detail, and by December 1956January 1957 reported to Marshak that there were internal inconsistencies or contradictions among some reported results. 
(b) By early 1957, ECG and Marshak were convinced that the only possible form for a UFI was the V - A form: for each set of four-fermion fields $\psi_{1}, \psi_{2}, \psi_{3}, \psi_{4}$ the interaction had to have the form

$$
\mathcal{L} \sim g \bar{\psi}_{1} \gamma^{\mu}\left(1+\gamma_{5}\right) \psi_{2} \bar{\psi}_{3} \gamma_{\mu}\left(1+\gamma_{5}\right) \psi_{4}
$$

This implied maximal parity violation.

(c) However, they saw that there were four experiments, one published (in preprint form) and three yet to be published, disagreeing with the predictions of $\mathrm{V}-\mathrm{A}$. They were: (i) The Rustad-Ruby experiment on electronneutrino angular correlation in ${ }^{6} \mathrm{He}$ decay. (ii) The experiment by Lederman et al. on the sign of electron polarization in $\mu$ decay; results privately communicated to $\mathrm{P}$. T. Mathews. (iii) The Anderson-Lattes experiment on the frequency of the electron mode in $\pi$ decay; private communication from M. Gell-Mann. (iv) The Novey-Telegdi experiment on asymmetry in polarized neutron decay, private communication from B. Stech.

(d) The seventh Rochester Conference on High Energy Physics was to be held during 15-19 April 1957 at Rochester. Marshak was the originator of this series of conferences. However, even though he and ECG had in hand results (b) and (c) above, keeping in mind his own position, the fact that ECG was still a PhD student, and that he himself was to present another talk on the nucleonnucleon potential, Marshak did not provide an opportunity for ECG to present their results even briefly.

(e) Instead, Marshak requested P. T. Mathews from England, then visiting Rochester, to present the V-A results as remarks during one of the discussion periods, and Mathews agreed. This however he did not do, as in the review talks by Lee and $\mathrm{Mme} \mathrm{Wu}$, the message was that $\beta$-decay was probably $\mathrm{V}$ and $\mathrm{T}$, and all in all, the UFI idea would have to be abandoned.

(f) Marshak was to spend summer 1957 as a consultant at Rand Corporation in Santa Monica, California, as was Murray Gell-Mann (born 1929). Marshak requested GellMann to arrange a lunch meeting with himself, ECG and a few others. ECG described their V - A analysis and results, and found Gell-Mann most cordial and appreciative.

(g) Soon after, as preparation for a talk Marshak was to give at the Padua-Venice 'Conference on Mesons and Recently Discovered Particles' in September 1957, ECG and Marshak sent in an abstract on V - A, and on 16 September 1957 (ECG's 26th birthday) submitted their paper titled 'The Nature of the Four-Fermion Interaction'22. As a matter of publication ethics, Marshak decided the same results should not be submitted to two different places. As it happened, the publication of the Padua-Venice Proceedings was delayed until May 1958.

(h) In the meantime, on 16 September 1957 (just a coincidence?), Feynman and Gell-Mann submitted their paper titled 'Theory of the Fermi Interaction' to the Physical Review ${ }^{23}$. It was published on 1 January 1958, but their route to the $\mathrm{V}-\mathrm{A}$ structure was based on rather weak theoretical grounds.

(i) Probably to make up for lost time, Marshak took the initiative to submit a very short paper with ECG to the Physical Review on 10 January 1958, titled 'Chirality Invariance and the Universal Fermi Interaction, ${ }^{\text {24 }}$. It appeared in print on 1 March 1958, but in most physicists' minds the Feynman-Gell-Mann paper had priority.

This reconstruction of events is based on later recollections of both ECG and Marshak (see below). It was in this complex manner, by what may be called a 'comedy of errors' ending with tragic consequences for some, that ECG and Marshak lost the credit due to them as being the first to conceive of the $\mathrm{V}-\mathrm{A}$ idea. Over the next two years all four experiments listed by them were repeated, the results changed, and fell in line with the ' $\mathrm{V}-\mathrm{A}$ ' predictions.

It seems that at least Salam and Nambu (see earlier section on the 'US years') were aware of and sensitive to the ways these events unfolded. In 1985, ECG received the first Physics Prize of the Third World Academy of Sciences (TWAS) set up by Salam at Trieste, Italy in 1983. ECG has described the V - A story in several places: the TWAS Prize acceptance speech 'Origins of the universal theory of weak interactions'; an essay titled 'Midcentury adventures in particle physics' also from 1985; and in his Autobiographical Notes in the 2006 volume edited by Ranjit Nair.

At ECG's 60th birthday celebrations in 1991, Marshak gave an after-dinner talk titled 'The pain and the joy of a major scientific discovery, ${ }^{25}$. This is an amazing combination of eloquence, candour and humility. After recounting in full detail the events sketched above, he asks for ECG's forgiveness for his three 'Cardinal Blunders': points (d), (g) and (i) in our account above. He also comments on Gell-Mann, exchanges with Feynman, and with J. R. Oppenheimer (1904-1967), in very revealing ways:

(i) 'Gell-Mann claims in his 1983 Catalunya (Spain) talk on "Particle theory from S matrix to quarks" that, at the "summit" luncheon meeting, he mentioned the V-A theory as a possible "last stand"; however, that claim does not jibe with the recollections of the other four participants in the "summit" meeting ... Gell-Mann's Catalunya remarks also do not jibe with Feynman's account of the genesis of the Feynman-Gell-Mann version of V - A theory ...'

(ii) From a letter Feynman wrote to Marshak in 1985: '... I hope some day we can get this straightened out and give Sudarshan the credit for priority that he justly deserves ... these matters all vex me - and I wish I had not caused you and Sudarshan such discomfort. At any opportunity I shall try to set the record straight - as I have always done - but nobody believes me when I am serious.'

'Indeed, even gracious Dick Feynman, who faithfully stuck to the facts on the priority question, apparently never read our original Padua-Venice paper but only our short note. How else to explain his statement as "Summarizer" at the Neutrino 1974 Conference, in which he said: 
"We have a conventional theory of weak interactions invented by Marshak and Sudarshan, published by Feynman and Gell-Mann, and completed by Cabibbo - I call it the conventional theory of weak interactions - the one which is described as the V-A theory".'

(iii) At a December 1957 meeting in Princeton, '... Oppenheimer enthused about the new V-A theory of Feynman and Gell-Mann as I listened in stunned surprise ... Nine years later-shortly before his death-Oppie rediscovered our Padua-Venice paper and wrote me: "It is a beautiful paper and, for whatever good it is, even at this late date I read it with excitement and great pleasure",

Sadly, Marshak passed away soon after, in a drowning incident in Mexico, in December 1992.

The V-A theory was never recognized for a Nobel award, either for ECG and Marshak or for Feynman and Gell-Mann. (Neither, it should be remembered, did Fermi get a Nobel for his 1934 work.) It went on to become the basic first step, the crucial ingredient, for the unification of electromagnetism and weak interactions, achieved by Sheldon Lee Glashow (born 1932), Salam and Steven Weinberg (born 1933) by 1967-68. For this they shared the 1979 Nobel Prize. At the 2006 Conference in Austin for ECG's 75th birthday, Glashow said in a message:

'... my thesis advisor, Julian Schwinger, ... published a deeply flawed version of the universal theory of weak interactions... They (Marshak and Sudarshan) presented a comprehensive analysis of the weak interaction data, which along with the imposition of an elegant symmetry principle, allowed them to deduce a unique form for the weak interactions, the so-called V - A theory.... The Sudarshan-Marshak paper was submitted practically at the same time as the Feynman-Gell-Mann paper, "Theory of the Fermi Interaction." ... However, several reasons underlie my belief that Sudarshan and Marshak deserve priority in this matter... . In my view, Sudarshan's seminal contribution to weak-interaction theory, representing only a small portion of his oeuvre, would itself justify the award of a major prize in Physics.'

It is appropriate to conclude this part of this section with a few sentences from Weinberg's talk ' $\mathrm{V}-\mathrm{A}$ was The Key' at Austin in 2006:

'... it took tremendous courage for Marshak and Sudarshan to propose ... that in fact the weak interaction was vector and axial vector, and not, as had been thought, scalar and tensor.... there really is a profound analogy between weak and electromagnetic interactions. It is not that the weak interactions are produced only by a vector current, as Fermi had thought; it certainly is not that weak interactions are produced by scalar and tensor interactions which had dominated the thinking of nuclear physicists for so many years; rather, weak interactions are produced by both vector and axial vector currents, as with great courage and physical insight and elan, Marshak and Sudarshan were the first to propose in 1957.'

\section{The case of the 'diagonal representation' in quantum optics}

The University of Rochester has a long and rich history in the field of optics - its Institute of Optics was set up in 1929. The iconic names of Kodak, Xerox and of Bausch and Lomb are of companies founded in Rochester. For many decades the emphasis was on classical optics, and then in the 1960s the quantum optics period began.

In 1959, when ECG came back from Harvard to a faculty position at Rochester, the first edition of the monumental book Principles of Optics by Max Born (18821970) and Emil Wolf was published ${ }^{26}$; and Wolf moved from Manchester in England to Rochester as a faculty member. From the very beginning, ECG and Wolf had a very cordial relationship. At that time Leonard Mandel (1927-2001) was visiting Rochester from Imperial College in London. Then in 1964, he too moved to a position at Rochester, just as ECG moved to Syracuse.

In the mid-1950s, Wolf had created the definitive formulation of classical optical coherence theory, treating the classical electromagnetic field as a stochastic process. The concept of partial coherence and its propagation laws were clarified - partial coherence was described by the so called 'two-point function', which was physically like the intensity but was propagated like an amplitude. This theory was included in Principles of Optics.

When the Hanbury-Brown-Twiss (H-B-T) effect was discovered in 1956 (ref. 27), initially there was considerable confusion about its conceptual basis, with some insisting that quantum ideas were essential to its understanding. Then in 1958, Mandel worked out a semiclassical treatment of photo-electron counting statistics ${ }^{28}$. Here the optical field incident on a photodetector is treated as a classical statistical system, belonging to an ensemble and experiencing fluctuations. The quantum mechanical photodetector acts as the apparatus or measuring device. From the statistics of the photocounter in time, one tries to reconstruct (at least partially) the statistical properties of the incident classical beam, thus passing from a quantum apparatus to a classical observed system! This work of Mandel helped in understanding the $\mathrm{H}-\mathrm{B}-\mathrm{T}$ effect as well - conceptually it is the result of intensity-intensity correlations in a fluctuating classical light beam.

Soon after this, the invention of the laser in 1960 led to the need for a well-formulated framework for quantum optics. Of course, the quantization of the Maxwell field had been done by Dirac in 1927, and reviewed by Fermi in 1932 (ref. 16). A manifestly covariant (and renormalizable) form of QED had been developed in the 1940s. 
For the most part, though, these methods had been used for few-photon processes - Compton, Møller, Bhabha, ... scattering. While in principle the quantization of the Maxwell field was completely known, what were needed were practical ways to describe quantum states 'close' to classical beams of light; and in particular the quantum counterpart of the new Born-Wolf classical theory of partial coherence needed to be developed. It was this that was achieved starting in 1963. The two key persons involved were R. J. Glauber and ECG.

In his theory of partial coherence in classical optics, Wolf had used the complex analytic signal, the positive frequency part $E^{(+)}(x)$ of, say, the electric field vector, introduced by Denis Gabor (1900-1979) in 1946 (ref. 29). Basic to the treatment of Young-type interference and diffraction phenomena was the two-point coherence or correlation function

$$
\Gamma^{(1,1)}\left(x ; x^{\prime}\right)=\left\langle E^{(-)}(x) E^{(+)}\left(x^{\prime}\right)\right\rangle, E^{(-)}(x)=E^{(+)}(x)^{*},
$$

with the angular brackets denoting averaging over a classical statistical ensemble of solutions of the Maxwell equations. (For simplicity we omit here suffixes on the $E$ s denoting the Cartesian vector components). To begin with, it is this that was generalized to the quantum case by Glauber.

At this point, as with the $\mathrm{V}-\mathrm{A}$ story, here too there have been conflicting claims of priority, and who did what and when; so it is useful to list in chronological sequence the four basic papers involved:

(a) Glauber - 'Photon Correlations' - Phys. Rev. Lett., submitted 27 December 1962, published 1 February 1963, 10, 84

(b) Glauber - 'The Quantum Theory of Optical Coherence'-Phys. Rev., submitted 11 February 1963, published 15 June 1963, 130, 2529.

(c) ECG - 'Equivalence of Semiclassical and Quantum Mechanical Descriptions of Statistical Light Beams' Phys. Rev. Lett., submitted 1 March 1963, published 1 April 1963, 10, 277.

(d) Glauber - 'Coherent and Incoherent States of the Radiation Field'-Phys. Rev., submitted 29 April 1963, published 15 September 1963, 131, 2766.

Paper (c) was ECG's first one in the field of optics.

In paper (a), Glauber generalized the classical definition (eq. (8)) to quantum theory using the annihilation and creation parts $\hat{E}^{(+)}(x), \hat{E}^{(-)}(x)$ of the quantized electric field operator:

$$
\begin{aligned}
& G^{(1,1)}\left(x ; x^{\prime}\right)=\operatorname{Tr}\left(\hat{\rho} \hat{E}^{(-)}(x) \hat{E}^{(+)}\left(x^{\prime}\right)\right), \hat{E}^{(-)}(x)=\hat{E}^{(+)}(x)^{+}, \\
& \hat{\rho}=\text { density matrix of state of quantized field. }
\end{aligned}
$$

Just as the classical treatment of the $\mathrm{H}-\mathrm{B}-\mathrm{T}$ effect involves the higher order correlation function

$$
\Gamma^{(2,2)}\left(x, y ; x^{\prime}, y^{\prime}\right)=\left\langle E^{(-)}(x) E^{(-)}(y) E^{(+)}\left(x^{\prime}\right) E^{(+)}\left(y^{\prime}\right)\right\rangle,
$$

Glauber also introduced the higher order quantum object

$$
G^{(2,2)}\left(x ; y ; x^{\prime}, y^{\prime}\right)=\operatorname{Tr}\left(\hat{\rho} \hat{E}^{(-)}(x) \hat{E}^{(-)}(y) \hat{E}^{(+)}\left(x^{\prime}\right) \hat{E}^{(+)}\left(y^{\prime}\right)\right) .
$$

(Note that in both eqs (9) and (11), the operators on the right are in normal-ordered form: creation operators to the left of annihilation operators.) He pointed out the importance of using the so-called coherent states of the photon field in these problems, and also noted that for a thermal state, $\hat{\rho}$ can be written as a convex (integral) combination of projections onto them. Since the properties of these states are crucial for what follows, we recall their definition and properties at this stage.

For simplicity we consider a single mode of the quantized radiation field, so all the photons are similar and are in that mode. The photon annihilation and creation operators, $\hat{a}$ and $\hat{a}^{+}$, obey the canonical commutation relation

$$
\left[\hat{a}, \hat{a}^{+}\right]=1 .
$$

The states of the single mode field make up a Hilbert space $\mathcal{H}$. The Fock states are those with definite numbers of photons, and they form an orthonormal basis (ONB) in $\mathcal{H}$. Starting from the no-photon state $|0\rangle$ we have

$$
\begin{aligned}
& n=0,1,2, \ldots:|n\rangle=\left(\hat{a}^{+}\right)^{n}|0\rangle / \sqrt{n !}, \\
& \hat{a}^{\dagger} \hat{a}|n\rangle=n|n\rangle,\left\langle n^{\prime} \mid n\right\rangle=\delta_{n^{\prime}, n}, \\
& \sum_{n=0}^{\infty}|n\rangle\langle n|=1 \text { on } \mathcal{H} .
\end{aligned}
$$

The last equation expresses completeness of these states. Thus the Fock states are the eigenstates of the Hermitian photon number operator $\hat{a}^{\dagger} \hat{a}$.

On the other hand, the coherent states $|z\rangle$ where $z$ is any complex number, are right eigenstates of the nonhermitian annihilation operator $\hat{a}$. Their expansions in the Fock basis and key properties are

$$
\begin{aligned}
& z \in \mathbb{C}:|z\rangle=\exp \left(-\frac{1}{2}|z|^{2}\right) \sum_{n=0}^{\infty} \frac{z^{n}}{\sqrt{n !}}|n\rangle, \\
& \hat{a}|z\rangle=z|z\rangle, \\
& \left\langle z^{\prime} \mid z\right\rangle=\exp \left(-\frac{1}{2}\left|z^{\prime}-z\right|^{2}+i \operatorname{Im} z^{\prime *} z\right) .
\end{aligned}
$$

Thus, they are always nonorthogonal. However, as shown by Klauder in 1960, they furnish a resolution of the identity $^{30}$

$$
\frac{1}{\pi} \int \mathrm{d}^{2} z|z\rangle\langle z|=1 \text { on } \mathcal{H}, \mathrm{d}^{2} z=d \operatorname{Re} z \mathrm{~d} \operatorname{Im} z
$$


In fact, they form an overcomplete set: any $|\psi\rangle \in \mathcal{H}$ can be expanded in terms of $\{|z\rangle\}$ in infinitely many ways, one of which is obtained by applying both sides of eq. (15) to $|\psi\rangle$.

Now we return to recounting the events in 1963. As ECG recollected later, sometime in February 1963, Wolf returned from a Conference in Paris - The Third International Congress on Quantum Electronics - where he had listened to Glauber's presentation of the ideas contained in paper (a). He felt discouraged that the quantum mechanical treatment would be beyond him, and conveyed this to ECG. The latter then reassured him that he would re-express and explain all that was involved in such a way that Wolf would understand everything with no difficulty. In this way, in double quick time, ECG did the calculations leading to this surprising result: on account of the overcompleteness of the coherent states $\{|z\rangle\}$, any density matrix $\hat{\rho}$ can be expressed in the 'diagonal representation' form

$$
\hat{\rho}=\frac{1}{\pi} \int \mathrm{d}^{2} z \phi(z)|z\rangle\langle z|,
$$

in terms of a suitable unique c-number diagonal weight $\phi(z)$. ECG even produced a formal (very singular) expression for $\phi(z)$ in terms of the Fock states matrix elements of $\hat{\rho}$ :

$$
\begin{aligned}
\phi(z)= & \sum_{n, n^{\prime}=0}^{\infty} \frac{\sqrt{n ! n^{\prime} !}}{\left(n+n^{\prime}\right) !}\left\langle n|\hat{\rho}| n^{\prime}\right\rangle \frac{\exp \left(r^{2}+i\left(n^{\prime}-n\right) \theta\right)}{2 \pi r} \\
& \times\left(\frac{-\partial}{\partial r}\right)^{n+n^{\prime}} \delta(r), \quad z=r \exp (i \theta) .
\end{aligned}
$$

Thanks to the normal ordering in eqs (9), (11) and (14), we then see that the quantum mechanical correlation functions take up the forms (in the single mode case).

$$
\begin{aligned}
& G^{(1,1)} \rightarrow \operatorname{Tr}\left(\hat{\rho} \hat{a}^{\dagger} \hat{a}\right)=\frac{1}{\pi} \int \mathrm{d}^{2} z \phi(z) z^{*} z, \\
& G^{(2,2)} \rightarrow \operatorname{Tr}\left(\hat{\rho}\left(\hat{a}^{\dagger}\right)^{2}(\hat{a})^{2}\right)=\frac{1}{\pi} \int \mathrm{d}^{2} z \phi(z) z^{* 2} z^{2},
\end{aligned}
$$

formally exactly as in a classical statistical ensemble but with $\phi(z)$ in place of a classical probability distribution.

ECG recalled that upon seeing all this, Wolf insisted that ECG draft a paper based on his calculations immediately, and send it off by priority mail for publication, and only then go for lunch. It is in this way that paper (c) in the Physical Review Letters came to be written. The result that, thanks to ECG's diagonal representation eq. (16), the quantum mechanical correlation functions defined by Glauber take up the classical looking forms of eq. (18) is called the 'optical equivalence theorem'.

At the November 2006 symposium in Austin, Texas for ECG's 75th birthday, the session on 'Quest 4: Quantum
Coherence' had presentations by three speakers: Chandra Lal Mehta (born 1938), Harry Jeffrey Kimble (born 1949) and Rajiah Simon (born 1948) (written version co-authored with M. D. Srinivas). Kimble emphasized the key role played by ECG's 'diagonal representation' in these words:

'... the Optical Equivalence Theorem has provided the central tool in Quantum Optics for distinguishing between classical and manifestly quantum (or nonclassical) regimes for the electromagnetic field. ... the function $\phi(z)$ is, in modern terms, called the GlauberSudarshan phase-space function. The aspect of this function that George emphasized (and formally established) is its universal character for all states of the electromagnetic field, ranging from black body radiation... to more pathological examples, such as a number (Fock) state ... the Optical Equivalence Theorem provides a definitive, model independent line of demarcation between the classical and manifestly quantum domains.'

He then went on to the exploration in the laboratory of the consequences of the Optical Equivalence Theorem, and to the creation and study of 'nonclassical light' corresponding to $\phi(z)$ not being a classical probability distribution.

The presentations by Mehta and Simon contain careful and detailed comparisons of the contents of Glauber's papers (a), (b) and (d) contrasted with ECG's paper (c). We summarize the main conclusions as follows:

(i) In paper (a), while the usefulness of the coherent states $|z\rangle$ is mentioned, there seems to be no appreciation of their overcompleteness, and no references to the fundamental mathematical results of Bargmann, Segal and Schweber used and quoted by $\mathrm{ECG}^{31}$.

(ii) Paper (a) contains the easily obtainable double integral representation (in the ECG notation!)

$$
\hat{\rho}=\iint \mathrm{d}^{2} z \mathrm{~d}^{2} z^{\prime} F\left(z, z^{\prime}\right)|z\rangle\left\langle z^{\prime}\right|,
$$

but for given $\hat{\rho}$ the function $F\left(z, z^{\prime}\right)$ is 'doubly nonunique', whereas the quantity $\phi(z)$ in eq. (16) is unique. Therefore, no clear distinction between classical and nonclassical states is possible on the basis of Glauber's eq. (19).

(iii) In paper (d), starting from his non-unique representation eq. (19), Glauber says: ‘... for a broad class of radiation fields which includes ... virtually all of those studied in optics, it becomes possible to reduce the density operator to a considerably simpler form ...'

This 'simpler form' is given by Glauber as

$$
\hat{\rho}=\int P(\alpha)|\alpha\rangle\langle\alpha| \mathrm{d}^{2} \alpha,
$$

which is just ECG's eq. (16) with the variable changes $z \rightarrow \alpha, \phi \rightarrow P$. Then a footnote is added: 
'the existence of this form for the density operator has also been observed by E. C. G. Sudarshan'. As Simon says, the word 'observed' should have been replaced by 'discovered', and this 'way of citing an earlier published paper ... is extraordinary, to say the least.'

(iv) In paper (d), there is no derivation of eq. (20) from eq. (19), no inversion formula for $P(\alpha)$ in terms of $\hat{\rho}$, and no clear statement of the states $\hat{\rho}$ for which the representation eq. (20) is claimed. From later remarks, one infers that Glauber accepts the validity of the representation eq. (20) only for states $\hat{\rho}$ which are classical by the diagonal representation criterion.

In view of all this, it is indeed strange that all too often the ECG Diagonal Representation (eq. (16)) is called, after a change of variables, the Glauber Representation or the Glauber-Sudarshan Representation.

In later joint work, Mehta and ECG exploited again the overcompleteness of coherent states to find other more convenient ways than eq. (17) to 'compute' $\phi(z)$ from $\hat{\rho}$, expressing its distribution theoretic nature in other ways $^{32}$. The book ${ }^{10}$ by Klauder and ECG gives a complete and thorough discussion of the nature of the diagonal weight $\phi(z)$, the extent of its singular nature for nonclassical states, and ways to approximate it by a sequence of tempered distributions.

Glauber shared the 2005 Nobel Prize with the experimentalists John L. Hall and Theodor W. Hansch. Simon's presentation shows how the Nobel citation and Glauber's Nobel lecture were both inaccurate and deeply flawed in the ways recounted above. No wonder that ECG expressed his pain in the Biblical quote: 'Give unto Glauber only what is his'.

For the work described in this section, ECG was nominated several times for the Nobel Prize in Physics. The fact that he was not so honoured was undoubtedly a source of deep unhappiness to him. In his 1985 essay 'Mid-century adventures in particle physics' he wrote:

'It has been a sad but wise experience to recognize that the universality of science does not imply unbiased acclaim for scientific truth and a true history of science; and that if you have neither powerful alliances nor influential sponsors you should learn to do science for its own sake and not be depressed by lack of appreciation. Over the years I have developed this skill...'

But the disappointment when the 2005 Nobel Prizes were announced was not alleviated by such an attitude. The only consolation may be that others too have had such experiences ${ }^{33}$.

We conclude this section with some remarks. It was mentioned that ECG's paper (c) was written (and then despatched) in an enormous hurry. It may have been wiser to have not rushed so much, as then sentences like these may have been avoided:

'... statistical states of a quantized (electromagnetic) field have been considered recently, and a quantum mechanical definition of coherence functions of arbitrary order presented. It is the aim of this note to elaborate on this definition and to demonstrate its complete equivalence to the classical description as long as no non-linear effects are considered.'

'The demonstration above shows that any statistical state of the quantum mechanical system may be described by a classical probability distribution over a complex plane, provided all operators are written in the normal ordered form.'

These may convey the content of the optical equivalence theorem inaccurately, since $\phi(z)$ is not always a classical probability distribution.

\section{The Indian connection -1970 s and after}

All through the years of his academic life in USA from 1959 onwards - at Rochester, Syracuse and then Austin ECG maintained strong links with the Indian physics community, and more generally with Indian science. Among his many professional friends and close collaborators, the following may be particularly mentioned: P. M. Mathews, S. P. Pandya, S. N. Biswas (1926-2005), T. Pradhan (born 1929), V. Singh (born 1938) and J. V. Narlikar (born 1938). He also interacted with many others from different scientific backgrounds and other walks of life.

During the 1960s he made extended visits to IMSc, the Delhi University Summer Schools in elementary particle physics at Dalhousie and Udaipur, and to Delhi University itself. In 1970-71, he was Sir C. V. Raman Distinguished Professor at the University of Madras, hosted by P. M. Mathews.

Probably ECG's most substantive involvement with Indian science began in 1971-72. Satish Dhawan (19202001), Director of IISc from 1962 to 1981, had just returned from a sabbatical year at the California Institute of Technology, USA. He was India's most distinguished fluid dynamicist and aeronautical engineer, who placed India's space programme on secure and strong foundations. Daulat Singh Kothari (1905-1993) was at that time Chairman of the University Grants Commission of India, a position he occupied from 1961 to 1973. Earlier he had been Professor of Physics at Delhi University (19341961), and the first Defence Science Advisor to the Government of India (1948-1961). As a result of initiatives taken by Dhawan and Kothari, and discussions among all three, ECG was invited to set up and direct a new Centre for Theoretical Studies (CTS) at IISc. This was to be 
concurrent with his responsibilities at the University of Texas at Austin, with him making two visits each year to IISc. The major aims and activities of CTS were to establish a place where any scientific problem with significant mathematical structure could be discussed and pursued; to organize seminars and conferences covering a wide range of disciplines, and to run a vibrant Visiting Scientists Programme especially for faculty and students from Indian universities and institutions.

The initial faculty put together in $1972-73$ by ECG with guidance from Dhawan consisted of Roddam Narasimha (born 1933), aeronautical engineering and fluid dynamics; Krityunjai Prasad Sinha (born 1929), theoretical physics; both already in IISc; N. Mukunda (born 1939), theoretical and mathematical physics; Madhav Gadgil (born 1942), evolutionary biology and ecology; Sulochana Gadgil (born 1944), atmospheric science and monsoon meteorology; and Hombegowda Sharat Chandra (born 1938), human genetics. A. K. Rajagopal (born 1938), theoretical physics, joined CTS for the year 1974 75. In 1976, Ramamurti Rajaraman (born 1939), Jagadisan Pasupathy (born 1940), both in theoretical physics; and Vidyanand Nanjundiah (born 1947), in evolution and developmental biology; came to CTS.

CTS functioned well in the early years, guided and inspired by ECG's vision. The Visiting Scientists Programme was probably the first of its kind in the country. Some of the particularly stimulating seminars were on 'The Mind: Pathways to its Understanding'; 'Science and Society'; 'Mathematical Models in Ecology'; and 'Human Genetics and Evolution'. In 1974, to celebrate the Golden Jubilee of Bose Statistics, a National Seminar on Statistical Physics was organized; and in 1982 a celebration of 25 years of the $\mathrm{V}-\mathrm{A}$ theory was held, to which Nambu was a distinguished invitee.

However, as the work of its diverse faculty grew, IISc created new specialized centres from out of CTS: the Centre for Atmospheric Sciences in 1982 (expanded in 1996 to include Oceanic Sciences); the Centre for Ecological Sciences in 1983, and the Developmental Biology and Genetics Laboratory in 2004. Newer faculty in CTS were largely in physics. Finally, in 2004, CTS was formally separated into a Centre for High Energy Physics, and a Centre for Contemporary Studies.

Sometime before these later changes at IISc, in 1984, ECG was invited to the Directorship of IMSc. He held this position till 1990, rejuvenating and expanding the scope of the Institute's activities. One of his important collaborators in this period, continuing from the early 1980s, was R. Simon. However, towards the end of this phase as Director of IMSc, the atmosphere was sadly vitiated on account of conflicts with some senior faculty members.

Up to about 1990, ECG pursued somewhat separate streams of activity at Austin, and in India. At Austin, the focus was largely on the Zeno problem in quantum mechanics, theory of open systems and their quantum dyna- mics, wave equations, and problems in quantum field theory. In India, the work was on refinements of the Diagonal Representation; group theoretical methods in problems of classical optics especially in dealing with partial coherence and polarization; the significance of the real symplectic groups $S p(2 n, R)$ for both optics and quantum mechanics, and geometric phase theory. In particular, ECG and some of his Indian collaborators exploited methods learnt from experience with quantum mechanics to pose and solve classical wave optical problems from a fresh perspective. In the period 1979-1984 he inspired and led a group of physicists from USA, Sweden, Italy and India collaborating on problems in mechanics, mathematical physics and topological ideas in elementary particle theory.

After about 1990, ECG's collaborations with physicists from Italy and Spain picked up in strength, the focus being mainly on quantum tomography, the Zeno effect and open system dynamics. The one to whom ECG was closest in outlook and interests was Giuseppe Marmo (born 1946) from Napoli.

ECG was honoured by the Government of India with the Padma Bhushan in 1976 and the Padma Vibhushan in 2007. He was elected to the Indian Academy of Sciences and the Indian National Science Academy in 1963 and 1972 respectively. In 1977, he delivered the Jawaharlal Nehru Memorial Lecture on 'Physics as a spiritual discipline', and also received the INSA S. N. Bose Medal. The prestigious Desikotthama Award of Visva Bharati came in 1998.

Among his recognitions and awards at the international level, the first TWAS Physics Prize in 1985 has been mentioned. In 1987, he was elected to the Academie Internationale de Philosophie des Sciences. In 2006, the birth centenary of Ettore Majorana (1906-1939), ECG received the first Majorana Prize. In 2010, the International Centre for Theoretical Physics, Trieste, Italy gave ECG and Nicola Cabibbo the Dirac Medal.

\section{Views on religion and philosophy - concluding remarks}

Apart from a few biographical details, this account has concentrated on ECG's life and work in physics and as a scientist. The complete list of his scientific publications and co-authored books are easily available to the interested reader. It is appropriate to conclude with a few words on other facets of his personality.

ECG was born into an orthodox Syrian Christian family, and was serious in his explorations of religion from quite a young age. In 2002, he made these statements about his attitudes ${ }^{34}$ :

'I was born in an Orthodox Christian family. I was very deeply immersed in it ... I was not quite satisfied with Christianity, and gradually I got more and more involved with traditional Indian ideas.' 
'I would now say I am a Vedantin, with these two religious and cultural streams mixed together.'

As a speaker, ECG had an entertaining style, a ready wit and plenty of humour. On the other hand, he was also a very intense person. In the early 1970s, he was attracted by the teachings of a Vedantin in Kerala. By about the mid to the late 1970s, he became close to Maharishi Mahesh Yogi and his institution in Switzerland. Each of these 'involvements' seems to have 'run out' after a few years. Again from the early 1970s or earlier, certainly right up to 1984 , he had several serious discussions with the philosopher Jiddu Krishnamurti, especially in Chennai and Bangalore. In many of his talks, ECG freely used Sanskrit verses and aphorisms - the 1977 Nehru Memorial Lecture and the 1985 TWAS Prize acceptance speech, for example, are full of them.

From about 2010, his health began to fail; much earlier a diabetic condition had been detected in 1984. The end came on 13 May 2018 in Austin, Texas. Bhamathi, a theoretical physicist, survives him.

\section{Selected references and footnotes}

1. Lee, T. D. and Yang, C. N., Question of parity conservation in weak interactions. Phys. Rev., 1956, 104, 254.

2. Wu, C. S., Ambler, E., Hayward, R. W., Hoppes, D. D. and Hudson, R. P., Phys. Rev., 1957, 105, 1413.

3. At just about the same time as the experiment by Wu et al. ${ }^{2}$, the conceptually simpler experiment using the decay $\Lambda \rightarrow p+\pi$ was done by Garwin, Lederman and Weinrich at Columbia University, and independently by Telegdi and Friedman at the University of Chicago, USA. The result of the former appeared in the same issue of the Physical Review ${ }^{2}$, while publication of the latter in the same journal seems to have been delayed because of a 'Columbia effect'.

4. Fermi, E., La Ricerca Scientifica, 1933, 4(2), 491; Attempt at a theory of $\beta$-rays, Il Nuovo Cimento, 1934, 11, 1; Z. Phys., 1934, 88, 161 .

5. Gamow, G. and Teller, E., Selection rules for the $\beta$-disintegration. Phys. Rev., 1936, 49, 895

6. Johnson, K. and Sudarshan, E. C. G., Inconsistency of the local field theory of charged spin 3/2 particles. Ann. Phys., 1961, 13, 126.

7. A detailed account of what happened may be found in Indira Chowdhury, Growing the Tree of Science - Homi Bhabha and the Tata Institute of Fundamental Research, Oxford University Press, 2016.

8. Sudarshan, E. C. G. and Mukunda, N., Classical Dynamics $-A$ Modern Perspective, Wiley, NY, USA, 1974; reprinted by Hindustan Book Agency, Delhi, 2015.

9. Currie, D. G., Jordan, T. F. and Sudarshan, E. C. G., Relativistic invariance and Hamiltonian theories of interacting particles. Rev. Mod. Phys., 1963, 35, 350.

10. Klauder, J. R. and Sudarshan, E. C. G., Fundamentals of Quantum Optics, W. A. Benjamin, New York, USA, 1968.

11. Marshak, R. E., Okubo, S. and Sudarshan, E. C. G., Consequences of charge independence for the magnetic moments and masses of $\Sigma$ hyperons. Phys. Rev., 1957, 106, 599.

12. Misra, B. and Sudarshan, E. C. G., The Zeno's paradox in quantum theory. J. Math. Phys., 1977, 18, 756.

13. Sudarshan, E. C. G., Mathews, P. M. and Rau, J., Stochastic dynamics of quantum-mechanical systems. Phys. Rev., 1961, 121, 920.

14. Chruscinski, D. and Pascazio, S., A brief history of the GKLS equation. Open Syst. Inf. Dyn., 2017, 24(3), 1740001.
15. It took ten years, from 1919 to 1929 , to establish that the electron energy spectrum was continuous. In the early 1920s, it seemed apparent that it was continuous except for some gamma-ray lines. Soon the continuity was clearly established by the work of C. D. Ellis and collaborators in England. But the outstanding experimentalist Lise Meitner (1878-1968) believed firmly in the spectrum being discrete, and did experiments to prove her claim. Ellis and co-workers did further experiments to support the continuous spectrum claim, but Meitner kept coming back with more data and arguments. Finally Ellis and Wooster did a calorimetric measurement which conclusively established the continuity of the spectrum; but Meitner would not agree until she had repeated the experiment herself. By now it was 1929.

16. Fermi, E., Quantum theory of radiation. Rev. Mod. Phys., 1932, 4, 87.

17. Yukawa, H., On the interaction of elementary particles I. Proc. Phys. Math. Soc. Jpn., 1935, 17, 48.

18. The $\mu$ meson was initially mistaken for that predicted by the Yukawa theory. It was only in a 1943 paper by S. Sakata and T. Inoue, published in 1946, that it was suggested that $\pi$ mesons are made by cosmic rays, followed by the sequence of decay processes $\pi^{-} \rightarrow \mu^{-}+\bar{v}_{\mu}$ and $\mu^{-} \rightarrow e^{-}+\bar{v}_{e}+v_{\mu}$. All these varieties of neutrinos were not known then, but they did suggest that $v_{\mu}$ is a spin $1 / 2$ neutral particle like $v_{e}$ but possibly different.

19. Klein, O., Nature, 1948, 161, 897; Tiomno, J. and Wheeler, J. A., Rev. Mod. Phys., 1949, 21, 153; Lee, T. D., Rosenbluth, M. N. and Yang, C. N., Phys. Rev., 1949, 75, 905.

20. Dallaporta, N., On the mean lives of heavy unstable particles. Il Nuovo Cimento, 1955, 1, 962.

21. Dalitz, R. H., Decay of $\tau$-mesons of known charge. Phys. Rev., 1954, 94, 1046.

22. Sudarshan, E. C. G. and Marshak, R. E., The Nature of the FourFermion Interaction. In Proceedings of the Conference on Mesons and Newly Discovered Particles, September 1957 (ed. Zanichelli, N.), Bologna, Padua-Venice, 1958. Reprinted in The Development of Weak Interaction Theory (ed. Kabir, P. K.), Gordon and Breach, 1963.

23. Feynman, R. P. and Gell-Mann, M., Theory of the Fermi Interaction. Phys. Rev., 1958, 109, 193.

24. Sudarshan, E. C. G. and Marshak, R. E., Chirality Invariance and the Universal Fermi Interaction. Phys. Rev., 1958, 109, 1860.

25. This is reprinted in the 2006 volume edited by Ranjit Nair mentioned in the section 'Events and publications from 2006'.

26. Born, M. and Wolf, E., Principles of Optics, Pergamon Press, Oxford, England, 1959, 1st edn.

27. Hanbury Brown, R. and Twiss, R. Q., Nature, 1956, 177, 27.

28. Mandel, L., Proc. Phys. Soc., 1958, 72, 1037.

29. Gabor, D., J. Inst. Elec. Eng. (London), 1946, 93, 429.

30. Klauder, J. R., Ann. Phys. (NY), 1960, 11, 123.

31. Bargmann, V., Commun. Pure Appl. Math., 1961, 14, 187; Segal, I. E., Illinois J. Math., 1962, 6, 500; S. S. Schweber, J. Math. Phys., 1962, 3, 831 .

32. Mehta, C. L. and Sudarshan, E. C. G., Phys. Rev., 1965, 138, B274.

33. Probably the most extreme case of this kind in physics is that of Arnold Sommerfeld (1868-1951). Between 1917 and 1951, he was nominated 84 times for the Nobel Prize, practically every year, but was never chosen. Among those who nominated him were Max Planck, Max von Laue, Wilhelm Wien, Robert Millikan, James Franck, and Enrico Fermi, all Nobelists. However, neither Albert Einstein nor Niels Bohr did so. Interestingly, none of his own 'Nobel' students - Werner Heisenberg, Wolfgang Pauli, Hans Bethe and Pieter Debye - ever nominated him.

34. George Sudarshan. In Science and the Spiritual Quest: New Essays by Leading Scientists (ed. Mark Richardson, W.), Routledge, 2002.

ACKNOWLEDGEMENTS. I thank Arvind, H. Sharat Chandra, Subhash Chaturvedi, G. Madhavan, Usha Mukunda, Sandip Pakvasa and Xerxes Tata for their comments and suggestions on a preliminary version of this article.

doi: $10.18520 / \mathrm{cs} / \mathrm{v} 116 / \mathrm{i} 2 / 179-192$ 